\title{
Diabetic nephropathy: What does the future hold?
}

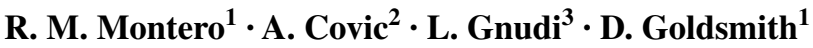

Received: 22 July 2015 / Accepted: 19 September 2015 / Published online: 5 October 2015

(C) The Author(s) 2015. This article is published with open access at Springerlink.com

\begin{abstract}
The consensus management of diabetic nephropathy (DN) in 2015 involves good control of glycaemia, dyslipidaemia and blood pressure (BP). Blockade of the renin-angiotensin-aldosterone system using angiotensin-converting enzyme inhibitors, angiotensin-2 receptor blockers or mineralocorticoid inhibitors are key therapeutic approaches, shown to be beneficial once overt nephropathy is manifest, as either, or both, of albuminuria and loss of glomerular filtration rate. Some significant additional clinical benefits in slowing the progression of DN was reported from the Remission clinic experience, where simultaneous intensive control of BP, tight glycaemic control, weight loss, exercise and smoking cessation were prioritised in the management of DN. This has not proved possible to translate to more conventional clinical settings. This review briefly looks over the history and limitations of current therapy from landmark papers and expert reviews, and following an extensive PubMed search identifies the most promising clinical biomarkers (both established and proposed). Many challenges need to be addressed urgently as in order to obtain novel therapies in the clinic; we also need
\end{abstract}

R. M. Montero

rosa_montero@hotmail.com

1 Renal, Dialysis and Transplantation Unit, Guy's and St Thomas' Hospital, London, UK

2 Hospital "C.I.Parhon" and University of Medicine "Grigore T Popa", Iasi, Romania

3 Cardiovascular Division, Department of Diabetes and Endocrinology, Guy's and St Thomas' Hospital, School of Medicine and Life Science, King's College London, London, UK to examine what we mean by remission, stability and progression of $\mathrm{DN}$ in the modern era.

Keywords Diabetic nephropathy $\cdot$ RAAS blockade . Albuminuria $\cdot$ Inflammation $\cdot$ Anti-fibrotics $\cdot$ New therapies

\section{Introduction: obesity, diabetes and CKD—a cardio-metabolic tsunami}

The rapidly rising incidence of diabetes mellitus (DM) worldwide is reputed to impact over 380 million people [1]. Table 1 shows the current and predicted incidences of chronic kidney disease (CKD), DM, and patients with DM that have reached end-stage kidney disease (ESKD). China now has the highest rates of number of people with DM, around 98.4 million, followed by India with 65.1 million and then the USA [2].

DM is the fifth cause of morbidity and mortality worldwide [3], and the most common cause of ESKD in the Western world [4]. The European Renal Association (ERAEDTA) registry data showed that ten countries across Europe had an increase of $11.9 \%$ per year of type 2 DM (T2DM) patients starting renal replacement therapy (RRT) [5].

T2DM increasingly arises in a younger and more obese population with metabolic syndrome [14] whose natural history is currently unknown but predicted to lead to full insulin resistance with a decline in renal function leading to CKD with proteinuria. The increasing rates of global obesity are a major drive in the development of diabetes, CKD and cardiovascular disease (CVD), representing a major health and health-economic burden to the developed and developing worlds. However, a significant proportion of patients with DM and CKD stage $3 \mathrm{a} / 3 \mathrm{~b}$ do not progress to ESKD and either stabilise with their current treatment 
Table 1 Predictions of population incidences of USA and UK [6-13]

\begin{tabular}{lll}
\hline Worldwide DM & $\begin{array}{l}1980-1990 \\
153 \text { million }\end{array}$ & $\begin{array}{l}2010-2020 \\
472 \text { million }\end{array}$ \\
\hline USA & & \\
CKD & 19 million & $>26$ million \\
DM & 5.8 million & 24 million \\
ESKD with DM & 17,727 & 48,215 \\
$U K$ & & \\
CKD & 1.7 million & 3.5 million \\
DM & 2.9 million & 5 million \\
ESKD with DM & 870,000 & 1.7 million \\
Europe & & \\
CKD & 59.3 million & 65.9 million \\
DM & 66.8 million & 68.9 million \\
ESKD with DM & 6.6 million & 6.8 million \\
\hline
\end{tabular}

therapies or die from their significantly higher cardiovascular mortality risks before RRT is needed [4, 15]. Analysis of the baseline characteristics of the Study of Heart and Renal Protection (SHARP) study showed the importance of primary renal disease leading to CKD [16]. The highest mortality overall is seen in patients with DM with a peak in mortality occurring prior to reaching ESKD.

The UK renal registry reported a mortality of $30 \%$ in DM on RRT aged 18-44 years at 5 years: much higher than the $11 \%$ observed in non-diabetic patients [17]. Following 5 years of RRT, $34 \%$ of non-diabetic patients aged 45-64 died compared with $51 \%$ in the DM group. The median life expectancy in non-diabetic patients requiring RRT aged $<45$ years was reported by the UK renal registry as 9 years more than in patients with DM of the same age. Thus, a further decade of life lost in this group of patients, many of whom, because of associated co-morbidities, are not promising candidates for pancreas and/or renal transplants.

Mahmoodi's meta-analysis of $>1$ million people showed an association between all-cause and cardiovascular mortality and CKD in non-hypertensive and hypertensive people with low GFRs and raised albumin-creatinine ratio (ACR). Patients with CKD alone had an all-cause and cardiovascular mortality, respectively, of 4.1 and $0.9 \%$, while those with CKD and hypertension had an all-cause and cardiovascular mortality, respectively, of 15 and $6.8 \%$. A subsequent meta-analysis looking at CKD with or without diabetes found similar relative risks of mortality between these two groups, thereby emphasising the importance of CKD as a major driver for mortality in these populations [18].

The degree of albuminuria has long been used to determine progression of DN with Adler reporting an annual incidence of people with diabetes progressing from normoalbuminuria to microalbuminuria $(2.0 \%$ year $)$ to macroalbuminuria (2.8\%/year) and to ESKD (2.3\%/year) [19]. This is otherwise known as the "classical progression paradigm", with mortality increasing at each stage (3.0, 4.6, 19.2\%/year, respectively). The decline in GFR has previously been thought to mirror changes in albuminuria in DN; however, there are increasing reports that the decline in GFR may occur irrespective of the degree of albuminuria in a non-proteinuric DN phenotype [20]. It is, however, well established that the presence of albuminuria and GFR independently and additively contribute to the cardiovascular and renal risk [21]. The alarming rates of disease incidence and progression continue despite the deployment of all current treatments and while achieving, in many cases, significant reductions in proteinuria require us to re-look at this well-known disease.

\section{The basic challenge: treating the whole patient by looking beyond renal endpoints}

\section{The pathophysiology, history, effects and limitations of RAAS blockade}

The mechanisms behind the pathophysiology of DN are complex and continue to be incompletely understood. Both metabolic (hyperglycaemia) and haemodynamic perturbations interact synergistically [22, 23] and have been reported to activate local RAAS resulting in increased angiotensin-2, reactive oxygen species (ROS), inflammation, expression of transforming growth factor- $\beta$ (TGF- $\beta$ ) and dysregulation of different vascular growth factors such as the VEGF-A (upregulated in the initial phase of the disease) and the angiopoietin/Tie-2 system (with excess of angiopoietin-2 over angiopoietin-1) [24] (Fig. 1). This would otherwise be known as the classical pathway of DN where the hallmark features of thickening of the glomerular basement membrane, mesangial expansion and glomerulosclerosis are classically seen on renal biopsy [25] in combination with albuminuria and GFR decline. Mauer had described these renal biopsy changes in 285 people with T1DM with normoalbuminuria who had been given enalapril, losartan or placebo. In this study, formal GFRs and a repeat renal biopsy were undertaken to determine any histological changes between those on different ACEi/ ARB treatments. This small study showed the decline in GFR and the change in mesangial fractional volume of each glomerulus determining the degree of mesangial expansion. They found the changes were comparable in all groups [26], and thus, albuminuria was insensitive to these changes.

The risk of renal biopsy has prevented further larger studies performing this procedure on those without proteinuria, and hence, it is unclear when the classical underlying 


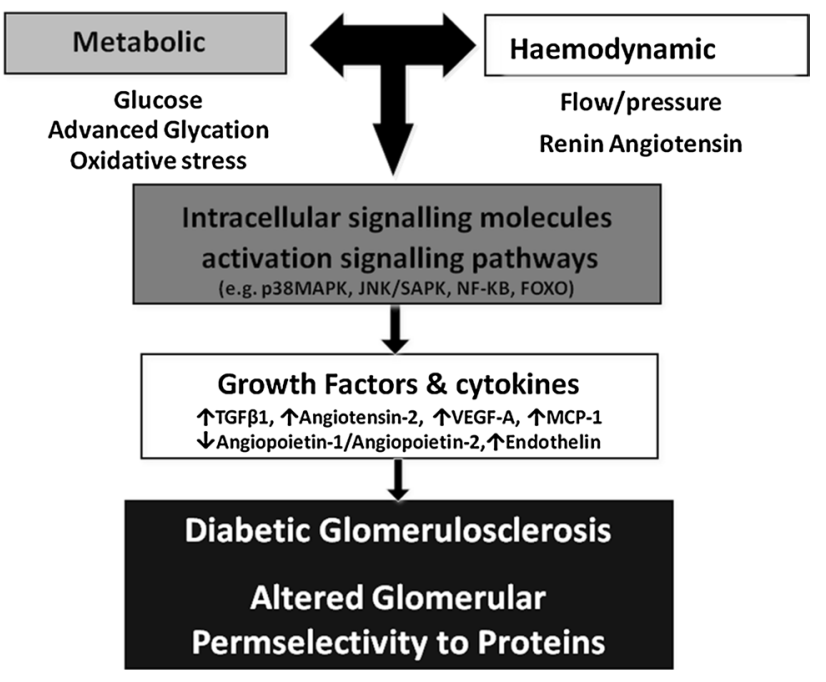

Fig. 1 Pathophysiology of DN

histological changes occur or alter with renal function decline or proteinuria. The success of a DN animal model is that which has developed these histological features and whereby treatments act to reverse them; however, the problem therein lies that the existing models of DN do not develop albuminuria or have this without the histological features seen in man. Interestingly, advanced renal damage has been reported in a study looking at nephrectomised cancer renal biopsies in those with T2DM without overt proteinuria suggesting that these changes may occur with GFR decline and worsen with the presence of proteinuria [20]. Other studies have attempted to reassess the features seen on the DN renal biopsy, and an increased heterogeneity within the histology of those with DN has been reported with the severity of glomerulopathy, tubulointerstitial fibrosis and arteriolar hyalinosis in those with microalbuminuria quantified in the Tervaert's histopathological classification $[25,27,28]$. This clearly illustrates that the simple paradigm of progression once depicted is far more complex than initially thought.

More recently, there has been appreciation for the role of inflammation in the progression of DN. The mechanisms employed are poorly understood with reports of an increase in inflammatory cytokines (MCP-1, TNF- $\alpha$ ) and mononuclear infiltrates in the glomeruli and tubulointerstitium that contribute to the progression of DN [29, 30]. Peroxisome proliferator-activated receptors- $\gamma$ (PPAR- $\gamma$ ) (glitazones) agonists have been reported to be reno-protective in experimental model of diabetes and in people with diabetes via their anti-inflammatory actions, in a metabolic independent manner [31, 32].

The RAAS has multiple roles in promoting progression of all renal diseases, particularly in DN [33]. The blockade of RAAS in animal and in vitro studies have shown a down-regulation of TGF- $\beta$, AGE, ROS, NADPH oxidase, RAGE expression and collagen IV (Col4) production-all leading to a reduction in mesangial expansion, glomerulosclerosis, inflammation and tubulointerstitial fibrosis [3439]. These findings have further fuelled the desire to block RAAS as the sole treatment for DN.

Numerous studies have demonstrated since the 1980s how good blood pressure (BP) and strict glycaemic control decrease cardiovascular mortality in patients with DM and renal impairment $[4,19,40]$. The treatment of patients with DM with CKD was revolutionised in the 1990s, with most cited landmark papers such as the Captopril study in type 1 DM (T1DM) reporting the loss of creatinine of $11 \%$ from $84 \mathrm{ml} / \mathrm{min}(\sim 9 \mathrm{ml} / \mathrm{min} /$ year $)$ versus $17 \%$ from $79 \mathrm{ml} / \mathrm{min}$ ( $\sim 13 \mathrm{ml} / \mathrm{min} /$ year) without captopril [41]. The Reduction of Endpoints in Non-insulin-dependent DM in the Angiotensin-2 Antagonist Losartan study (RENAAL) [42] simultaneously published with the Irbesartan Diabetic Nephropathy Trial (IDNT) both showed a decrease in GFR decline or a delay in the doubling of creatinine and a reduction in proteinuria in the ARB arm [43]. The possibility of preventing renal progression with eliminating this residual proteinuria with further agents suggested a further benefit by achieving complete RAAS blockade.

\section{Earlier stage DN: normoalbuminuria to proteinuria, microalbuminuria to proteinuria}

A delay in progression from microalbuminuria to overt proteinuria was described since 1995 with earlier studies focused on determining the significance of increasing proteinuria [44, 45]. Subsequently, Perkins showed factors associated with regression rather than progression of proteinuria. The DCCT/EDIC study showed $28 \%$ of microalbuminurics became macroalbuminuric with $15 \%$ sustaining a loss of GFR and $4 \%$ leading onto ESKD [46]. The introduction of RAAS blockade reduced proteinuria; regression rates to normoalbuminuria were $40 \%$, thus emphasising the relative insensitivity of albuminuria as a marker of progression in DN [47].

Undeniably, GFR decline occurs more rapidly in the presence of overt or nephrotic range proteinuria [48]; however, there has been a lack of appreciation of the degree of GFR decline occurring with minimal albuminuria. The loss of GFR is increasingly being reported in diabetic patients with normoalbuminuria, again illustrating how treatments targeting the resolution of albuminuria may already be too late in the disease or not work at all $[49,50]$. In addition, formulae used to determine GFR are not sensitive at levels of GFR $>90 \mathrm{ml} / \mathrm{min}$. The limitations of this formula are also apparent within the acute kidney injury arena where people with normal renal function without proteinuria undergo a substantial rapid decline in renal function 
secondary to an insult that may range from sepsis to drug induced that is not identified until the decline reaches levels the formula identifies as low [51]. The modification of diet in renal disease (MDRD) formula has been established as having a higher sensitivity in GFR $<60 \mathrm{ml} / \mathrm{min}$ with the chronic kidney disease epidemiology (CKD-EPI) formula reported to be more sensitive at higher GFRs; however, fundamentally the formulae are dependent on creatinine as the gold standard sensitive marker of renal injury. This marker, however, is non-specific to the type of histological injury. The laborious iohexol/inulin methods are employed in cases where true GFR is fundamental in providing drug treatments such as chemotherapy or for donors prior to living kidney donation [52] and may detect decline in renal function; however, these are seldom used to assess the renal function in those with DN.

The introduction of RAAS blockade at this early stage of DN may not provide any benefit and emphasises the urgent need for more sensitive markers to detect early stage DN. This also challenges the simple paradigm of albuminuria leading to GFR loss and steady progression that may be conceptually too simplistic for such a complex disease.

\section{Combining RAAS blockade with other, mainly cardioprotective, interventions}

Dual blockade of RAAS was done using different agents in a number of studies with differing effects on the surrogate endpoint trials have adopted (Table 2). The safety of dual blockade was brought into question in these studies where new therapies are additive to existing ACEi/ARB therapy. The use of this combination in T2DM patients continues to be controversial and currently is not recommended. The effects of aliskiren alone have not been assessed as trials conducted with new agents currently act as adjuncts to RAAS blockade rather than alternatives to ACEi/ARB.

\section{Intensification of therapies with RAAS blockade}

The importance of a multistrategy approach with RAAS blockade led to the Steno-2 study [56], whereby intensive glycaemic and BP control was achieved. Smoking cessation, weight loss and a reduction in dietary sodium intake were additionally maintained. The results supported a multifactorial approach $[56,57]$ to achieve lower cardiovascular (CV) events and mortality in the intensely monitored group after 13.3 years [58]. A multimodal strategy approach to the management of DN is supported by the Remission clinic [59]. Dual RAAS inhibition was employed with intensified BP control, statin use, smoking cessation and implementation of a healthy lifestyle. The median followup was 4 years and suggested that a multidrug approach in the treatment of CKD could slow the progression of CKD in T2DM and non-diabetics, similar to the Steno-2 study [56]. The Remission clinics had a median decline of -0.17 versus $-0.56 \mathrm{ml} / \mathrm{min} / 1.73 \mathrm{~m}^{2} /$ year of GFR in the intensive versus conventional treatment groups. BP decreased and a $50 \%$ decline in urinary proteinuria from baseline levels was seen. Intensive monitoring avoided a rise in hyperkalaemic events supporting the use of this treatment strategy in T2DM with hypertension and normoalbuminuria, similar to the Bergamo Nephrologic Diabetes Complications Trial (BENEDICT); however, all the patients in the BENEDICT trial were hypertensive to begin with [60]. The BENEDICT trial initially showed that trandolapril delayed the onset of microalbuminuria in T2DM with normoalbuminuria and hypertension.

Previous studies suggest a reduction in $\mathrm{CV}$ endpoints and mortality in all patients with diabetes adhering to a diastolic BP of $<80 \mathrm{mmHg}$ [61-63]. Whether a systolic BP of $<130$ or $<140 \mathrm{mmHg}$ has additional benefits on CVD and mortality remains unknown [61]. Systolic BP $<125 \mathrm{mmHg}$ was reported to be deleterious in the MDRD study and in diabetic patients $>70$ years of age [64-66]. To achieve systolic $\mathrm{BP}<130 \mathrm{mmHg}$ requires an intense approach seen in

Table 2 Outcome of landmark studies with RAAS blockade

\begin{tabular}{lllll}
\hline Trial & AVOID [53] & ALTITUDE [50] & VA NEPHRON D [54] & Aldosterone antagonists [55] \\
\hline Agent & Aliskiren & Aliskiren & ACEi (Lisinopril) & Aldosterone antagonist \\
Combined ACEi/ARB & ARB (Losartan) & ARB (Irbesartan) & ARB (Losartan) & ACEi/ARB (Lisinopril/Losartan) \\
Reduction of blood pressure & Yes & Yes & Yes & Yes \\
Decrease in the reduction in GFR & Yes $(-2.4 \mathrm{mls} / \mathrm{min})$ & Trial terminated & No effect & Yes (13\% less than placebo) \\
Anti-proteinuric effect & Yes & Yes & Yes & Yes \\
Effect on CV mortality & No effect & Increased events & No effect & Not powered \\
Hyperkalaemic events & Same as placebo & Increased & Increased & Increased \\
Acute kidney injury & Same as placebo & Increased & Increased & Same as placebo \\
Progression to ESKD & No effect & Trial terminated & No effect & Not powered \\
Number of patients & 599 & 8561 & 1448 & 81 \\
\hline
\end{tabular}


Table 3 Current renal endpoints used in trials

\begin{tabular}{llll}
\hline Trial & Primary renal endpoints & Effects on ACR & Effects on GFR \\
\hline $\begin{array}{l}\text { PREVEND (Prevention of Renal and Vascu- } \\
\text { lar End-stage Disease Intervention trial) }\end{array}$ & $\begin{array}{l}\text { CV mortality, hospitalisation for CV mor- } \\
\text { bidity and reaching ESKD }\end{array}$ & Decrease & No change \\
RENAAL (see above) & CV mortality, time to ESKD & Decrease & Decrease GFR slowed with ARB \\
IDNT (see above) & Double serum creatinine & Decrease & Decrease GFR slowed with ARB \\
BEACON (see below) & Decrease risk of ESKD or CV mortality in & Increase & Increase in GFR \\
& T2DM with CKD IV & & \\
\hline
\end{tabular}

Table 4 Biomarkers for DN

\begin{tabular}{ll}
\hline Mechanism & Potential biomarkers \\
\hline Glomerular & Cystatin C \\
Tubuloepithelial & Urinary Col4, transferring, caeruloplasmin, MCP-1 \\
Oxidative stress and inflammation & NAG, NGAL, KIM-1 \\
& Urinary $\alpha$ 1-microglobulin, L-FABP, CTGF, RBP \\
Endothelial dysfunction & Urinary 8-OHdG \\
& Serum TNFR, IL-6, TNF $\alpha$ \\
Genomics, proteomics and metabolomics & Urinary pentosidine, TNF $\alpha$, acid glycoprotein, VEGF, YKL-40 \\
microRNAs & Tyrosine kinase \\
\hline
\end{tabular}

both the Remission and Steno-2 single-centre approaches. Despite considerable efforts, few patients managed smoking cessation or regular exercise in the Steno-2 study. The Steno-2 study also supplemented glycaemic and BP control with dietary supplements of vitamins $\mathrm{E}$ and $\mathrm{C}$ together with folic acid, which is not standard clinical practice. These approaches, however, (Steno, Bergamo) are completely impractical for normal clinical practice due to the intensity required to manage the needs of an ever-growing population.

\section{The next challenge: defining renal endpoints and how to measure them}

Traditionally, a change in proteinuria, a doubling of serum creatinine or reaching ESKD are the renal endpoints used to determine outcome and efficacy of treatment in renal trials (Table 3).

These traditional "biomarkers" have several limitations (see above) and require large and costly interventional trials. The trial endpoints currently used require long trials to assess the $\mathrm{CV}$ mortality and whether a progression of ESKD or a double of serum creatinine is reached in this variably progressive disease. Using these trial endpoints limits, the ability to determine treatment effect with no insight into how the natural history of the disease and its complications are affected, due to the longer time point surrogate markers employed. This has led to suggestions of measuring other proteins in new clinical trials to compare this with albuminuria [50]. To assess whether the change or persistence in albuminuria continues despite the new intervention and compares these effects with the different severity of disease within the chosen population. It has also been suggested that an alternative surrogate endpoint could be a 30-40 \% decrease in GFR with albuminuria defined as new onset, progression or regression with micro-/macroalbuminuria, however, discussion in this area continue [67]. Development of new biomarkers looking at different underlying structural damage or pathophysiological pathways opens new avenues for assessing progressive DN (Table 4); however, how these biomarkers correlate with human histopathological changes is currently unknown and not routinely practiced.

\section{Biomarkers in DN: Better than albuminuria or GFR?}

The limitations of using albuminuria or GFR alone are apparent with their use as trial endpoints requiring further thought. New biomarkers also have their limitations and may be perceived as no better than those established, however, using more specific markers that are specific to 
underlying glomerular or tubular dysfunction, the different stages of disease progression may be identified and used to eloquently individualise therapies.

\section{Glomerular damage}

Cystatin $\mathrm{C}$ is a $13.3-\mathrm{kDa}$ freely filtered plasma protein that is catabolised by tubular cells, thereby not returning to the circulation and has been described as a more sensitive marker of renal function than serum creatinine in early DN $[68,69]$. This marker could be adopted for trials looking at early DN where GFR is less sensitive.

Urinary and serum Col4 with a low molecular weight (LMW) of $540 \mathrm{kDa}$ are elevated in DN [70]. Raised urinary Col4 levels occur in normoalbuminuric DN and could be an earlier marker than microalbuminuria of underlying glomerular damage [71]. However, Col4 levels decrease with improved glycaemic control, limiting its use [72]. In contrast, urinary transferrin $(76.5 \mathrm{kDa})$ is a plasma protein that increases independently of albuminuria [73] and a predictor of development of microalbuminuria in normoalbuminuric T2DM irrespective of BP or HbA1c [74]. This may be a useful marker in CKD 3a disease or to determine underlying hyperfiltration.

Urinary monocyte chemoattractant protein-1 (MCP-1/ CCL2) increases in macroalbuminuric but not in microalbuminuria T2DM patients [75]. Urinary MCP-1 levels have been shown to correlate with $\mathrm{DN}$ tubulointerstitial lesions, fibrosis and glomerular injury [76, 77]. This biomarker has the potential to determine progressors, and in trials looking at microalbuminuria, detection of this could be used as a new endpoint of predicting ongoing disease. Raised urinary caeruloplasmin $(132 \mathrm{kDa})$ is an acute-phase protein whose excretion increases in normoalbuminuric $\mathrm{DN}$, but its usefulness is limited by its reversibility with improved glycaemic control [78]. Matix metalloproteinases (MMP9) are reported to be increased in DN where the ECM has broken down and there is increased cell turnover. MMP9 is also raised during podocyte dedifferentiation and may be used as an early marker of DN [79].

\section{Tubuloepithelial makers}

Trials measuring biomarkers with different underlying histopathologies will help develop a greater understanding of the occurrence of these processes and landmark disease processes. This will allow treatments to be specifically targeted to the underlying pathways leading to these changes and allow them to be adapted and personalise the individuals, therapy to prevent progression. Tubuloepithelial markers in combination with glomerular markers may result in a reasonable predictive trial surrogate endpoint.
An increase in secretion or decrease in reabsorption of LMW plasma proteins may be detected in the urine reflecting tubuloepithelial cell damage. $N$-acetyl- $\beta$-Dglucosaminidase (NAG) is a $140-\mathrm{kDa}$ lysosomal enzyme in renal tubules that increases in normoalbuminuric DN with significantly raised levels occurring with transition from micro- to macroalbuminuria in T2DM [80]. Neutrophil gelatinase-associated lipocalin (NGAL) (25-kDa) protein stored in neutrophilic granules bind and transport small hydrophobic molecules to regulate the immune system and apoptosis [81]. Raised levels of NGAL are seen in the hyperfiltration stage of DN with a lowering of GFR and a raised cystatin $\mathrm{C}$ when compared with healthy controls [82]. When adjusted for BP, HbAlc and duration of diabetes, the difference is no longer significant. Kidney injury molecule-1 (KIM-1) is a type-1 cell membrane glycoprotein on the apical membrane of the proximal tubule that increases with tubular damage in DN [83]. Hyper-filtration in T2DM results in raised urinary levels of KIM-1; however, these are decreased with RAAS blockade limiting its use to a marker of active tubular injury [84].

Urinary connective tissue growth factor (CTGF) and TGF- $\beta$ are increased in micro- and macroalbuminuric DN $[85,86]$ and may reflect the occurrence of underlying fibrosis that may be used as a marker for disease progression; $15-\mathrm{kDa}$ intracellular carrier protein liver-type fatty acid binding protein (L-FABP) found in the liver and proximal tubular cells is a marker of structural and functional renal tubular damage. This increases with diabetes and albuminuria [87]. Again, its use is limited with the use of RAAS blockade. In contrast, urinary retinal binding protein (RBP), a $21-\mathrm{kDa}$ freely filtered microprotein that is almost completely reabsorbed in the proximal tubules, is a highly sensitive marker of early DN with its excretion reflecting damage in normoalbuminuria [88]. RBP has also been correlated with micro- and macrovascular complications of T2DM. Studies suggest urinary $\alpha 1$-microglobulin (A1M), a 26-kDa microprotein similar to RBP, may be used to determine tubular dysfunction in early DN [89].

\section{Markers of oxidative stress and inflammation}

ROS are produced with hyperglycaemia and contribute to diabetic complications via the accumulation of sorbitol and the formation of advanced glycation end products (AGE) and activation of protein kinase $\mathrm{C}$ pathways. Enzyme cleavage of ROS-induced 8-hydroxylation of guanine base from nuclear and mitochondrial DNA results in 8-oxo-7, 8-dihydro-2-deoxyguanosine $(8-\mathrm{OHdG})$ as a product of oxidative DNA damage in the urine [90]. These urinary levels may be used to determine early underlying oxidative stress damage. 
Serum and urinary TNF $\alpha$ levels increase with micro- and microalbuminuria in T2DM [91]. TNF receptors (TNFRs) 1 and 2 mediate the effects of TNF $\alpha$. Serum levels of these membrane bound receptors correlate with GFR in DN independent of albuminuria [92]. Raised baseline serum TNFR in patients with DM has predicted progression of CKD 3-5 independently of albuminuria after 12 years [93, 94] and is a promising biomarker of early DN.

Serum interleukin-6 (IL-6) increases with albuminuria and in those with underlying glomerular basement membrane thickening $[95,96]$. Urinary orosomucoid or $\alpha 1$ acid glycoprotein (AGA) is a polypeptide released in response to inflammatory cytokines IL-6 and TNF $\alpha$ and reported to be raised in CVD, lung cancer and DM [97]. Urinary levels of vascular endothelial growth factor (VEGF) (an angiogenic inducing cytokine related to endothelial permeability) are increased in normoalbuminuric DN [98].

\section{Endothelial dysfunction}

YKL-40 is a pro-inflammatory marker independently associated with albuminuria in early T2DM [99, 100]. High levels have been reported to predict CV mortality [101]. Tyrosine kinase is predominantly found in endothelial cells and has been described as a biomarker for underlying angiogenesis pathways [102]. It binds to angiopoietin of endothelial cells to induce angiogenesis. C-type natriuretic peptide (CNP) arises from damage to the vascular endothelium and is a predictor of GFR decline [103].

\section{Genomics, proteomics and metabolomics}

The development of DN arises from a combination of genetic and environmental factors. Genome-wide association scans have been widely used to determine gene variants predisposing to DN [104]. AFF3 gene on chromosome 15 has been described in T1DM with DN and also seen as an inducer of TGF- $\beta$ fibrosis in cultured epithelial cells [105]. By contrast, proteomics will provide multiple markers of DN combining cytokines, collagen fragments and RBP [106-108]. A panel of 273 urinary peptides (CKD273) was reported to be specific and sensitive for the early detection of DN [109]. The risk of progression was identified with this panel in a cohort of 35 patients with DM and normoalbuminuria followed up for 5 years [110]. CKD273 has subsequently been validated in a multicentre study that distinguished DN from controls with a factor cut-off, thereby determining individuals at a high risk of progressing [110, 111].

There is a paucity of studies in metabolomics whereby the measure of cellular function using LMW intermediate and end products of the cell reflect the genomic, transcription and proteomic function of the cell affected by the underlying disease pathology [112]. Changes have been detected in the phospholipid and amino acid metabolism of normoalbuminuric diabetic patients and healthy controls [113]. Consideration of the benefit of renal biopsy in trial patients to assess the effect of treatment and that of disease on the biomarkers used may need to be taken prior to determining the accuracy of these markers to reflect underlying disease processes in man.

\section{MicroRNAs}

MicroRNAs (miR) are short non-coding RNAs of 20-22 nucleotide length that may be measured in plasma and urine and use posttranscriptional mechanisms to result in gene silencing via the reduction of translation or induction of target degradation [114, 115]; $60 \%$ of the human protein coding genes are thought to be regulated by $\mathrm{miR}$ [116]. miR192 upregulates miR216a/217 and miR200b/c that increases collagen expression stimulated by TGF- $\beta$ miRmediated mechanism. This may promote autoregulation of TGF- $\beta 1$ seen in mouse mesangial cells and renal glomeruli of mouse models of DN [117, 118]. Mice with deletion of miR192 in vivo were protected from DN [119]. miR192 promoter has also been seen to be regulated by Smads via histone acetylation via TGF- $\beta$ in renal cells $[120,121]$. The downregulation of miR29 correlated with an increase in collagens I, III and IV in ApoE-/- diabetic mouse kidneys in proximal tubuloepithelial cells, mesangial cells, podocytes and TGF- $\beta$. miR192 promotes fibrosis with miR29 being anti-fibrotic [120, 122]. A single study, however, showed in patients with diabetes a decrease in miR192 was associated with increased severity of fibrosis and DN; however, this study did not determine normal levels of miR192 in healthy fibrosis [123].

The miRs levels vary from cell type to cell type, and thus, the overall levels in a condition need to be determined before this can be used as a tool to predict progression of DN. Urinary miR levels have been measured together with circulating levels in patients with CKD and may prove to be useful biomarkers of disease [124]. Potential antimiR target drugs with conventional therapy may be a new approach in the management of DN.

Recently, Pena et al. [103] published an observational study looking at the value of a panel of 28 serum biomarkers on predicting a decline in GFR in 82 patients with a 4-year follow-up. The two statistical models determined a change of $<3 \mathrm{ml} / \mathrm{min} / 1.73 \mathrm{~m}^{2} /$ year with the chosen biomarkers reflecting different pathways previously reported to be involved in DN. Other studies have used urinary biomarkers [125] or a combination of plasma and urinary biomarkers [126]; however, these panels have not been validated or used in a mixed population of T1/T2DM with varying degrees of albuminuria. 
Another approach to the selection of biomarkers has recently been reported by Lambers Heerspink et al. [127]. The SysKid consortium performed a comparison of systematic analysis of molecular pathways for DN previously described in the literature with a systematic analysis of the molecular pathways and mode of action of a drug of interest, such as ACEi. The overlapping molecular pathways were used to identify biomarkers similar to both and provide a basis to study subgroups and identify these as phenotype profiling. This novel approach may identify within-groups genetic polymorphisms that determine whether a specific group within the DN spectrum will have a more effective response to the therapy and thereby enhance personalised therapies to precision medicine. This data analysis combines genomic, proteomic, metabolomic and transcriptomic molecular pathways and closely centres around the effects of new therapies to provide biomarkers and maybe useful when monitoring the treatment effect of certain therapies in the future once validated.

The pathogenesis of DN is increasingly accepted as involving multiple mechanisms [24], and in response, there are now a host of novel approaches to DN (Table 5). New therapeutic strategies are emerging with some currently being conducted in small trials with some promising initial results [128-131].

\section{Novel interventions to tackle the problem}

\section{Antioxidants and anti-inflammatories}

Oxidative stress and inflammation have been increasingly described to be active in the progression of CKD, in particular in DN [128, 132, 133]. Nuclear-1-factor (erythroidderived 2)-related factor 2 ( $\mathrm{Nrf} 2$ ) is a transcription factor driving the antioxidant response, impaired in experimental animal models of CKD [134]. Bardoxolone methyl is a synthetic triterpenoid that activates the Nrf2 pathway, restoring antioxidant response. Early human studies with T2DM and CKD stage $3 \mathrm{~b}-4$ reported reductions in serum creatinine concentration with bardoxolone methyl for up to 52 weeks $[135,136]$. Subsequently, the BEACON trial was designed to determine whether bardoxolone methyl reduced the risk of ESKD or cardiovascular mortality in T2DM with CKD stage 4 [137]. A significant increase in GFR, BP, body weight and urinary ACR was seen in the bardoxolone methyl compared with the placebo group. However, the BEACON trial was terminated following a median follow-up of 9 months after 43 patients receiving bardoxolone methyl developed ESKD and increased cardiovascular morbidity and mortality (from any cause) were observed in the intervention arm when compared to placebo, with "heart failure" being a prominent signal. It is postulated that the side effects arose due to a longer exposure period to the drug in individuals with a more severe form of CKD; however, further detailed analysis is pending. There were suggestions that bardoxolone was toxic in mouse models of DN that were not translated to the initial human study although the improvement in GFR in the initial study remains unexplained [138]. Novel Nrf2 activators are in the pipeline, and future studies will determine their potential benefit in the clinical setting [139-141].

Smaller studies have looked at other targets to determine whether there is any benefit to combined treatment with RAAS blockade (Table 5). Pyridoxamine (vitamin B6) has been shown in animal studies to be a natural inhibitor of AGE with additional reductions in albuminuria and preservation of renal function [142, 143]. Lewis et al. [144] conducted a randomised double-blind placebo-controlled trial in T2DM treated with pyridoxamine for a year, but the results were negative.

Pentoxifylline is a methylxanthine derivative with inhibitory actions against TNF- $\alpha$. The PREDIAN trial shows significant reductions in proteinuria $(p<0.01)$ and preservation of renal function with pentoxifylline in combination with ACEi/ARB therapy compared to ACEi/ARB therapy alone $(p<0.001)$ in CKD stages 3/4 and T2DM [145].

Silymarin is a herbal drug with antioxidant, anti-inflammatory and anti-fibrotic properties. It is the active constituent of the seed Silybum marianum, otherwise known as milk thistle. In a small study, silymarin reduced albuminuria, urinary $\mathrm{TNF} \alpha$ and malondialdehyde - a marker of oxidative stress in patients with DN [146].

Macrophage recruitment into adipose tissue is thought to contribute to insulin resistance. The development of a CCR2/MCP-1 antagonist in animal studies was accompanied by better glycaemic control while decreasing glomerulosclerosis and albuminuria in diabetic mice [147]. More recently, the results of a randomised placebo-controlled study in $89 \mathrm{~T} 2 \mathrm{DM}$ normoalbuminuric patients were given the CCR2 antagonist has shown improved glycaemic control while being generally well tolerated [148].

\section{Anti-fibrotics}

Endothelin-1 has established pro-fibrotic effects within the kidney [149] following activation of endothelin-1A receptors leading to renal cell injury, inflammation and fibrosis. Endothelin is raised in CKD with endothelin receptor antagonists reported to ameliorate these effects in experimental models of CKD [150]. Endothelin-1A antagonist (atrasentan) with ACEi/ARB significantly reduced albuminuria in a recent randomised controlled trial in T2DM; however, heart failure and fluid overload were the main side effects limiting this treatment [151]. A novel endothelin antagonist (avosentant) is currently been tested for renal and cardiovascular endpoint in patients with T2DM and proteinuria as add-on to RAAS inhibition. 


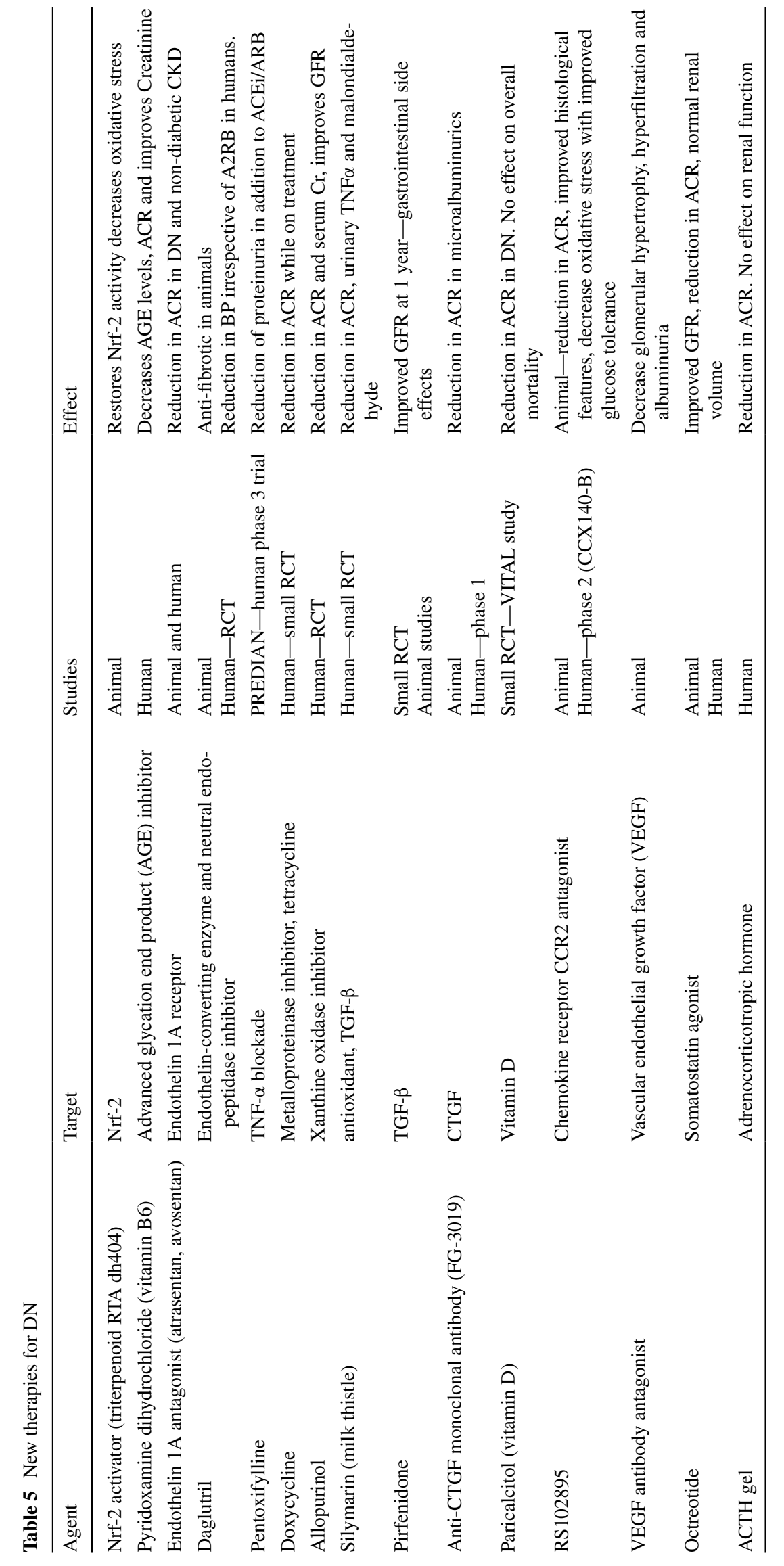


Daglutril is a combination between endothelin-converting enzyme and neutral endopeptidase inhibitor drugs with potent anti-hypertensive actions that are synergistic with RAAS blockade [152]. Minimal facial and peripheral oedema was thought to arise from the inhibitions of the neutral endopeptidase that increase natriuretic peptides, bradykinin and substance P. This 8 -week study showed effects on BP alone. Longer studies will determine any potential renoprotective effect of this drug in diabetes.

The anti-fibrotic pirfenidone inhibits TGF- $\beta$ in animal models [131]. A small trial showed an improvement in GFR at 1 year, but gastrointestinal symptoms and fatigue were common in this small study [153]. Interestingly, the benefit in GFR was seen using a lower rather than a higher dose of pirfenidone and further studies are required. CTGF has been shown to have an important effect on the development of renal fibrosis in DN [154]. The antiCTGF monoclonal antibody has been given to 24 microalbuminuric T1DM or T2DM with the effect of reducing albuminuria up to a year later; however, a larger trial is pending [130]. Recently, anti-TGF- $\beta 1$ therapy failed to slow disease progression in patients with advanced DN in a randomised, double-masked, phase 2 dose-ranging study [155].

Vitamin D deficiency has been shown in animal studies to contribute to the severity of albuminuria, podocyte effacement and increased glomerular basement membrane [156]. Replacement with an active analogue reduces proteinuria, interstitial fibrosis while improving renal function [157, 158]. A meta-analysis of vitamin D analogues in humans has shown a reduction of proteinuria in combination with RAAS blockade using an ACEi/ARB [159].

An increase in the expression of VEGF has been described in renal biopsies with $\mathrm{DN}$ and is thought to contribute to albuminuria and glomerulosclerosis $[4,34]$. Antibodies against VEGF have shown a reduction in the histological features of DN in diabetic rats with a decrease in albuminuria [160], and may be a potential future treatment.

\section{Anti-proteinurics}

Octreotide is a somatostatin analogue that decreases mesangial expansion and albuminuria in DM animal models [161]. A small study showed an improvement in GFR, ACR and no change in renal biopsy volume in T1DM given octreotide [162], with a further study showing a reduction of albuminuria following 6 months of treatment [163].

Recently, subcutaneous adrenocorticotrophic hormone gel was given to patients with DN with nephrotic range proteinuria; this treatment achieved a significant reduction in proteinuria after 6-12 months which continued for a year following cessation of treatment. Renal function remained stable in these studies [164].
Doxycycline is a tetracycline antibiotic that inhibits matrix metalloproteinases (MMPs). A decrease in proteinuria was seen at 6 months in combination with RAAS blockade and anti-hyperglycaemic agents in a small cohort [165]. The cessation of doxycycline resulted in the return of previous levels of albuminuria. Allopurinol used in asymptomatic hyper-uricaemic patients with DN was seen to reduce albuminuria in 4 months of treatment in this small RCT of 40 patients and could be used as an adjunct with RAAS blockade [166]. More recently, the PERL is underway recruiting patients with T1DM and CKD stages 1-3 to be given allopurinol for a 3-year period, and the results are awaited [167]. However, allopurinol has recently been reported in T2DM to significantly decrease serum uric acid, ACR, serum creatinine and improve GFR compared to the conventional treated arm [168].

\section{Current limitations and challenges of regulatory requirements for study of late-stage $\mathrm{DN}$}

The old adage ignorance is bliss may be applied with the current belief that there is adequate understanding in the field of the pathogenesis of this disease that has been described since Egyptian times [169] and continues to afflict millions. The major limitation with studies in this field fundamentally lies around the lack of a good biomarker to determine disease stage and without establishing the pathophysiology of this disease to a higher-degree primary prevention, and early intervention trials are made incredibly difficult. Establishing whether glomerular processes versus tubular damage versus ROS/other pathway activation occur first will more effectively allow new emerging therapies to be personalised to the individual with monitoring, using a panel of more sensitive markers reflecting underlying damage or activation of established pathways. Further validation of biomarkers arising from the comparison of molecular pathways of certain DN phenotypes whose genetic polymorphisms may make them more susceptible to certain drug therapies may provide a platform for precision medicine in the future.

The emergence of new biomarkers should be considered to run in parallel with albuminuria in trials allowing for increased sensitivity and comparison to the classical 'normo-, micro- and macroalbuminuria' while we increase our understanding. Subsequently, biomarkers that have been discovered depicting early or later stages should be employed to allow treatment effects to be determined. The biomarkers should be used to assess the different CKD stages occurring within those with DN and arguably the change of GFR may be a better comparator for new biomarkers. The treatments should then be used to target the area the biomarker suggests is the most likely active cause 
of damage. Mann et al. [170] recently refer to the benefit of using a roadmap for studies involving a number of different stakeholders such as clinicians, statisticians, patient groups, health insurance and pharmaceutical companies, biobanks and regulatory agencies, to help avoid delays in the discovery and introduction of therapies and biomarkers to the clinic.

Without the roadmap approach, funding inevitably limits long-term studies and emphasises the importance of multicentre trials whereby large groups of people with the same CKD stage can be compared. The additional challenge is that of the different rates of progression affecting those with DN that may be in part influenced by whether patients are T1DM or T2DM. The final common pathway of fibrosis affects both; however, it is well established that the autoimmune T1DM differs from the metabolic phenotype of those with T2DM and insulin resistance rather than autoimmune destruction of the pancreatic $\beta$-islet cells. It would seem more prudent to continue to separate trials for type of diabetes or to conduct trials with a subgroup analysis of T1DM and T2DM within the CKD stages to ascertain whether the results and biomarkers behave in the same manner or differ, thereby making markers more robust while more importantly allowing clarity as to what population benefits from a particular new therapy.

Where does this leave the researcher?-to investigate the pathophysiology and markers of disease to determine new therapeutic targets with this understanding. Great attention to differences seen in translational treatments is important in view of the recent experience with bardoxolone.

Where does this leave the clinician?- to be aware of changes occurring in the field and to actively enrol appropriate patients into trials where new therapies are being monitored with more sensitive markers. Both researcher and clinician need to urgently review the current primary endpoints of: doubling of serum creatinine, ESKD or death used in this chronic disease especially with early intervention trials. For trials conducted in late-stage DN, all patients should be monitored in specialised DN clinics. In addition, clinicians need to establish within their countries health service infrastructure how patients may be followed up with either dedicated DN clinics or primary care facilities to record how effective therapies are following the completion of initial funding for the trial period. We would challenge the cessation of follow-up for these patients until we are able to determine outcome and would advocate a national database for DN. Larger multicentre trials will naturally enhance our understanding in this field and should be increasingly considered in view of the worldwide increasing incidence of this devastating disease.

More importantly, where does this leave the patients?in a new era, an awakening leading to personalised treatment goals according to the progression or stability of their disease with the hope of preventing progression to ESKD.

\section{Final conclusions and future strategies for clinical research}

RAAS blockade alone is insufficient in preventing the progression of diabetic kidney disease in many patients. Good glycaemic control is important in reducing mortality in this cohort of patients; however, very tight glycaemic control may be deleterious [171]. No benefits have been reported for aspirin in the primary prevention of cardiovascular mortality in patients with diabetes; however, it is recommended in those who have had a cardiovascular event. Statins may reduce major cardiovascular events without affecting the progression of renal disease.

There are clear and urgent needs to improve our understanding of the pathogenesis of DN, identify new interventional therapeutic targets and develop new markers of disease progression. The new targets for intervention need to be tested using a melange of old and new markers of disease progression to maximise the chance of detecting useful biological signals. New therapeutics will become available presenting the challenge of when to begin these treatments and how to tailor treatments to the individual. Some of these agents such as vitamin B6, pentoxifylline and vitamin D are readily available and may be introduced to current therapy strategies. Whether any of these novel approaches can usefully reduce mortality remains to be determined.

This review emphasises the need for physicians to look anew at treatment strategies for this high-risk group and perhaps employ the use of multiple treatment strategies beyond that of RAAS blockade. We challenge the current renal endpoints used and how we continue to conduct trials. The need for new therapeutic agents, coupled with refined and more sophisticated trial renal endpoints using new biomarkers, is both obvious and urgent requiring our community to reunite in order to advance treatment strategies in this field.

\section{Compliance with ethical standards}

Conflict of interest None of the authors have any conflict of interest.

Open Access This article is distributed under the terms of the Creative Commons Attribution 4.0 International License (http://creativecommons.org/licenses/by/4.0/), which permits unrestricted use, distribution, and reproduction in any medium, provided you give appropriate credit to the original author(s) and the source, provide a link to the Creative Commons license, and indicate if changes were made. 


\section{References}

1. Danaei G et al (2011) National, regional, and global trends in fasting plasma glucose and diabetes prevalence since 1980: systematic analysis of health examination surveys and epidemiological studies with 370 country-years and 2.7 million participants. Lancet 378(9785):31-40

2. $\mathrm{Xu} \mathrm{Y}$ et al (2013) Prevalence and control of diabetes in Chinese adults. JAMA 310(9):948-959

3. Roglic $\mathrm{G}$ et al (2005) The burden of mortality attributable to diabetes: realistic estimates for the year 2000. Diabetes Care 28(9):2130-2135

4. Dronavalli S, Duka I, Bakris GL (2008) The pathogenesis of diabetic nephropathy. Nat Clin Pract Endocrinol Metab 4(8):444-452

5. Van Dijk PC et al (2005) Renal replacement therapy for diabetic end-stage renal disease: data from 10 registries in Europe (1991-2000). Kidney Int 67(4):1489-1499

6. Burrows NR, Li Y, Geiss LS (2010) Incidence of treatment for end-stage renal disease among individuals with diabetes in the U.S. continues to decline. Diabetes Care 33(1):73-77

7. Coresh J et al (2003) Prevalence of chronic kidney disease and decreased kidney function in the adult US population: Third National Health and Nutrition Examination Survey. Am J Kidney Dis 41(1): 1-12

8. Collins AJ et al (2003) Chronic kidney disease and cardiovascular disease in the Medicare population. Kidney Int Suppl 87:S24-S31

9. The DIAMOND Project Group (2006) Incidence and trends of childhood Type 1 diabetes worldwide 1990-1999. Diabet Med 23(8):857-866

10. Onkamo $\mathrm{P}$ et al (1999) Worldwide increase in incidence of Type I diabetes - the analysis of the data on published incidence trends. Diabetologia 42(12):1395-1403

11. Ruggenenti P, Remuzzi G (2007) Kidney failure stabilizes after a two-decade increase: impact on global (renal and cardiovascular) health. Clin J Am Soc Nephrol 2(1):146-150

12. Diabetes, W.E. (2014) WHO/Europe Diabetes, www.euro.who. int/en/health-topics

13. Care, N.k. (2010) NHS kidney care 2010. www.healthcheck.nhs.uk

14. Fagot-Campagna A et al (2000) Type 2 diabetes among North American children and adolescents: an epidemiologic review and a public health perspective. J Pediatr 136(5):664-672

15. de Boer MJ, Bilo HJ (2014) Clinical decision making and cardiovascular risk factors: what about the Heart Team? Rev Clin Esp 214(4):200-201

16. Haynes R et al (2014) Evaluating the contribution of the cause of kidney disease to prognosis in CKD: results from the Study of Heart and Renal Protection (SHARP). Am J Kidney Dis 64(1):40-48

17. Association, R (2013) 16th annual report, Dec 2013. https:// www.renalreg.org/reports/2013-the-sixteenth-annual-report/

18. Mahmoodi BKet al (2013) Association of chronic kidney disease with adverse outcomes—authors' reply. Lancet 381(9866):532-533

19. Adler AI et al (2003) Development and progression of nephropathy in type 2 diabetes: the United Kingdom Prospective Diabetes Study (UKPDS 64). Kidney Int 63(1):225-232

20. Porrini E et al (2015) Non-proteinuric pathways in loss of renal function in patients with type 2 diabetes. Lancet Diabetes Endocrinol 3(5):382-391

21. Ruggenenti P et al (2012) Measurable urinary albumin predicts cardiovascular risk among normoalbuminuric patients with type 2 diabetes. J Am Soc Nephrol 23(10):1717-1724

22. Cooper ME (2001) Interaction of metabolic and haemodynamic factors in mediating experimental diabetic nephropathy. Diabetologia 44(11):1957-1972
23. King GL (2008) The role of inflammatory cytokines in diabetes and its complications. J Periodontol 79(8 Suppl):1527-1534

24. Gnudi L (2012) Cellular and molecular mechanisms of diabetic glomerulopathy. Nephrol Dial Transplant 27(7):2642-2649

25. Fioretto P, Caramori ML, Mauer M (2008) The kidney in diabetes: dynamic pathways of injury and repair. The Camillo Golgi Lecture 2007. Diabetologia 51(8):1347-1355

26. Mauer M et al (2009) Renal and retinal effects of enalapril and losartan in type 1 diabetes. N Engl J Med 361(1):40-51

27. Tervaert TW et al (2010) Pathologic classification of diabetic nephropathy. J Am Soc Nephrol 21(4):556-563

28. Fioretto $P$ et al (1996) Patterns of renal injury in NIDDM patients with microalbuminuria. Diabetologia 39(12):1569-1576

29. Navarro-Gonzalez JF et al (2011) Inflammatory molecules and pathways in the pathogenesis of diabetic nephropathy. Nat Rev Nephrol 7(6):327-340

30. Gnudi L, Thomas SM, Viberti G (2007) Mechanical forces in diabetic kidney disease: a trigger for impaired glucose metabolism. J Am Soc Nephrol 18(8):2226-2232

31. Yang $\mathbf{J}$ et al (2009) Role of PPARgamma in renoprotection in Type 2 diabetes: molecular mechanisms and therapeutic potential. Clin Sci (Lond) 116(1):17-26

32. Setti G et al (2010) Peroxisome proliferator-activated receptor-gamma agonist rosiglitazone prevents albuminuria but not glomerulosclerosis in experimental diabetes. Am J Nephrol 32(5):393-402

33. Furukawa $M$ et al (2013) Pathogenesis and novel treatment from the mouse model of type 2 diabetic nephropathy. ScientificWorldJournal 2013:928197

34. Yamagishi S, Matsui T (2010) Advanced glycation end products, oxidative stress and diabetic nephropathy. Oxid Med Cell Longev 3(2): 101-108

35. Liao J et al (2003) Effects of candesartan, an angiotensin II type 1 receptor blocker, on diabetic nephropathy in KK/Ta mice. $\mathrm{J}$ Nephrol 16(6):841-849

36. Fan Q et al (2004) Candesartan reduced advanced glycation end-products accumulation and diminished nitro-oxidative stress in type 2 diabetic KK/Ta mice. Nephrol Dial Transplant 19(12):3012-3020

37. Forbes JM et al (2002) Reduction of the accumulation of advanced glycation end products by ACE inhibition in experimental diabetic nephropathy. Diabetes 51(11):3274-3282

38. Mauer SM et al (1984) Structural-functional relationships in diabetic nephropathy. J Clin Invest 74(4):1143-1155

39. Doi $T$ et al (2008) The current clinical problems for early phase of diabetic nephropathy and approach for pathogenesis of diabetic nephropathy. Diabetes Res Clin Pract 82(Suppl 1):S21-S24

40. Parving $\mathrm{HH}$ et al (1983) Early aggressive antihypertensive treatment reduces rate of decline in kidney function in diabetic nephropathy. Lancet 1(8335):1175-1179

41. Lewis EJ et al (1993) The effect of angiotensin-convertingenzyme inhibition on diabetic nephropathy. The Collaborative Study Group. N Engl J Med 329(20):1456-1462

42. Brenner BM et al (2001) Effects of losartan on renal and cardiovascular outcomes in patients with type 2 diabetes and nephropathy. N Engl J Med 345(12):861-869

43. Lewis EJ et al (2001) Renoprotective effect of the angiotensinreceptor antagonist irbesartan in patients with nephropathy due to type 2 diabetes. N Engl J Med 345(12):851-860

44. The Diabetes Control and Complications (DCCT) Research Group (1995) Effect of intensive therapy on the development and progression of diabetic nephropathy in the Diabetes Control and Complications Trial. Kidney Int 47(6):1703-1720 
45. Warram JH et al (2000) Progression of microalbuminuria to proteinuria in type 1 diabetes: nonlinear relationship with hyperglycemia. Diabetes 49(1):94-100

46. de Boer IH et al (2011) Long-term renal outcomes of patients with type 1 diabetes mellitus and microalbuminuria: an analysis of the Diabetes Control and Complications Trial/Epidemiology of Diabetes Interventions and Complications cohort. Arch Intern Med 171(5):412-420

47. Perkins BA et al (2007) Microalbuminuria and the risk for early progressive renal function decline in type 1 diabetes. J Am Soc Nephrol 18(4):1353-1361

48. Ruggenenti P et al (2000) Chronic proteinuric nephropathies: outcomes and response to treatment in a prospective cohort of 352 patients with different patterns of renal injury. Am J Kidney Dis 35(6): 1155-1165

49. Krolewski AS et al (2014) Early progressive renal decline precedes the onset of microalbuminuria and its progression to macroalbuminuria. Diabetes Care 37(1):226-234

50. Levey AS et al (2009) Proteinuria as a surrogate outcome in CKD: report of a scientific workshop sponsored by the National Kidney Foundation and the US Food and Drug Administration. Am J Kidney Dis 54(2):205-226

51. Pickering JW, Endre ZH (2010) Back-calculating baseline creatinine with MDRD misclassifies acute kidney injury in the intensive care unit. Clin J Am Soc Nephrol 5(7):1165-1173

52. Stevens LA, Levey AS (2009) Measured GFR as a confirmatory test for estimated GFR. J Am Soc Nephrol 20(11):2305-2313

53. Parving $\mathrm{HH}$ et al (2008) Aliskiren combined with losar$\tan$ in type 2 diabetes and nephropathy. $\mathrm{N}$ Engl $\mathrm{J}$ Med 358(23):2433-2446

54. Fried LF et al (2013) Combined angiotensin inhibition for the treatment of diabetic nephropathy. $\mathrm{N}$ Engl $\mathrm{J}$ Med 369(20):1892-1903

55. Mehdi UF et al (2009) Addition of angiotensin receptor blockade or mineralocorticoid antagonism to maximal angiotensinconverting enzyme inhibition in diabetic nephropathy. J Am Soc Nephrol 20(12):2641-2650

56. Gaede $\mathrm{P}$ et al (2003) Multifactorial intervention and cardiovascular disease in patients with type 2 diabetes. $\mathrm{N}$ Engl $\mathrm{J}$ Med 348(5):383-393

57. Rachmani $\mathrm{R}$ et al (2002) Teaching patients to monitor their risk factors retards the progression of vascular complications in high-risk patients with Type 2 diabetes mellitus-a randomized prospective study. Diabet Med 19(5):385-392

58. Gaede P et al (2008) Effect of a multifactorial intervention on mortality in type 2 diabetes. N Engl J Med 358(6):580-591

59. Remission Clinic Task Force, Clinical Research Center "Aldo e Cele Dacco" (2011) The Remission Clinic approach to halt the progression of kidney disease. J Nephrol 24(3):274-281

60. Ruggenenti P et al (2011) Effects of verapamil added-on trandolapril therapy in hypertensive type 2 diabetes patients with microalbuminuria: the BENEDICT-B randomized trial. $\mathrm{J}$ Hypertens 29(2):207-216

61. Sarafidis PA, Ruilope LM (2014) Aggressive blood pressure reduction and renin-angiotensin system blockade in chronic kidney disease: time for re-evaluation? Kidney Int 85(3):536-546

62. UK Prospective Diabetes Study Group (1998) Tight blood pressure control and risk of macrovascular and microvascular complications in type 2 diabetes: UKPDS 38. BMJ 317(7160):703-713

63. Hansson L et al (1998) Effects of intensive blood-pressure lowering and low-dose aspirin in patients with hypertension: principal results of the Hypertension Optimal Treatment (HOT) randomised trial, HOT Study Group. Lancet 351(9118):1755-1762

64. Klahr S et al (1994) The effects of dietary protein restriction and blood-pressure control on the progression of chronic renal disease. Modification of Diet in Renal Disease Study Group. N Engl J Med 330(13):877-884

65. O'Hare AM et al (2009) Current guidelines for using angiotensin-converting enzyme inhibitors and angiotensin II-receptor antagonists in chronic kidney disease: is the evidence base relevant to older adults? Ann Intern Med 150(10):717-724

66. Sarafidis PA, Bakris GL (2009) Does evidence support reninangiotensin system blockade for slowing nephropathy progression in elderly persons? Ann Intern Med 150(10):731-733

67. Muskiet MH et al (2015) Pleiotropic effects of type 2 diabetes management strategies on renal risk factors. Lancet Diabetes Endocrinol 3(5):367-381

68. Ogawa $Y$ et al (2008) Serum cystatin $C$ in diabetic patients. Not only an indicator for renal dysfunction in patients with overt nephropathy but also a predictor for cardiovascular events in patients without nephropathy. Diabetes Res Clin Pract 79(2):357-361

69. Perkins BA et al (2005) Detection of renal function decline in patients with diabetes and normal or elevated GFR by serial measurements of serum cystatin $\mathrm{C}$ concentration: results of a 4-year follow-up study. J Am Soc Nephrol 16(5):1404-1412

70. Kado $S$ et al (1996) Urinary type IV collagen as a marker for early diabetic nephropathy. Diabetes Res Clin Pract 31(1-3):103-108

71. Cohen MP, Lautenslager GT, Shearman CW (2001) Increased collagen IV excretion in diabetes. A marker of compromised filtration function. Diabetes Care 24(5):914-918

72. Kotajima $\mathrm{N}$ et al (2000) Type IV collagen as an early marker for diabetic nephropathy in non-insulin-dependent diabetes mellitus. J Diabetes Complications 14(1):13-17

73. Narita $T$ et al (2004) Parallel increase in urinary excretion rates of immunoglobulin G, ceruloplasmin, transferrin, and orosomucoid in normoalbuminuric type 2 diabetic patients. Diabetes Care 27(5):1176-1181

74. Kazumi $T$ et al (1999) Increased urinary transferrin excretion predicts microalbuminuria in patients with type 2 diabetes. Diabetes Care 22(7):1176-1180

75. Tam FW et al (2009) Urinary monocyte chemoattractant protein-1 (MCP-1) and connective tissue growth factor (CCN2) as prognostic markers for progression of diabetic nephropathy. Cytokine 47(1):37-42

76. Wada $T$ et al (2000) Up-regulation of monocyte chemoattractant protein-1 in tubulointerstitial lesions of human diabetic nephropathy. Kidney Int 58(4):1492-1499

77. Banba N et al (2000) Possible relationship of monocyte chemoattractant protein-1 with diabetic nephropathy. Kidney Int 58(2):684-690

78. Yamazaki M et al (1995) Urinary excretion rate of ceruloplasmin in non-insulin-dependent diabetic patients with different stages of nephropathy. Eur J Endocrinol 132(6):681-687

79. Li SY et al (2014) Matrix metalloproteinase-9 deficiency attenuates diabetic nephropathy by modulation of podocyte functions and dedifferentiation. Kidney Int 86(2):358-369

80. Weitgasser R et al (1999) Prospective evaluation of urinary $\mathrm{N}$-acetyl-beta-D-glucosaminidase with respect to macrovascular disease in elderly type 2 diabetic patients. Diabetes Care 22(11):1882-1886

81. Yang YH et al (2009) Changes of serum and urine neutrophil gelatinase-associated lipocalin in type- 2 diabetic patients with nephropathy: one year observational follow-up study. Endocrine 36(1):45-51

82. Nielsen SE et al (2012) Tubular markers are associated with decline in kidney function in proteinuric type 2 diabetic patients. Diabetes Res Clin Pract 97(1):71-76

83. van Timmeren MM et al (2007) Tubular kidney injury molecule-1 (KIM-1) in human renal disease. J Pathol 212(2):209-217 
84. Nielsen SE et al (2012) The effect of RAAS blockade on markers of renal tubular damage in diabetic nephropathy: u-NGAL, u-KIM1 and u-LFABP. Scand J Clin Lab Invest 72(2):137-142

85. Sharma K, Ziyadeh FN (1997) Biochemical events and cytokine interactions linking glucose metabolism to the development of diabetic nephropathy. Semin Nephrol 17(2):80-92

86. Nguyen TQ et al (2006) Urinary connective tissue growth factor excretion correlates with clinical markers of renal disease in a large population of type 1 diabetic patients with diabetic nephropathy. Diabetes Care 29(1):83-88

87. Kamijo A et al (2004) Urinary excretion of fatty acid-binding protein reflects stress overload on the proximal tubules. Am J Pathol 165(4):1243-1255

88. Holm J, Nielsen NV, Hemmingsen L (1994) Retinopathy in type II diabetes mellitus associated with above-normal urinary excretion of RBP. Kidney Int Suppl 47:S105-S108

89. Hong CY et al (2003) Urinary alpha1-microglobulin as a marker of nephropathy in type 2 diabetic Asian subjects in Singapore. Diabetes Care 26(2):338-342

90. Cooke MS et al (2000) Urinary 8-oxo-2'-deoxyguanosine-source, significance and supplements. Free Radic Res 32(5):381-397

91. Navarro JF et al (2008) Influence of renal involvement on peripheral blood mononuclear cell expression behaviour of tumour necrosis factor-alpha and interleukin-6 in type 2 diabetic patients. Nephrol Dial Transplant 23(3):919-926

92. Niewczas MA et al (2009) Serum concentrations of markers of TNF-alpha and Fas-mediated pathways and renal function in nonproteinuric patients with type 1 diabetes. Clin J Am Soc Nephrol 4(1):62-70

93. Gohda $\mathrm{T}$ et al (2012) Circulating TNF receptors 1 and 2 predict stage $3 \mathrm{CKD}$ in type 1 diabetes. J Am Soc Nephrol 23(3):516-524

94. Niewczas MA et al (2012) Circulating TNF receptors 1 and 2 predict ESRD in type 2 diabetes. J Am Soc Nephrol 23(3):507-515

95. Moresco RN et al (2013) Diabetic nephropathy: traditional to proteomic markers. Clin Chim Acta 421:17-30

96. Dalla Vestra M (2005) Acute-phase markers of inflammation and glomerular structure in patients with type 2 diabetes. J Am Soc Nephrol 16(Suppl 1):S78-S82

97. Fournier T, Medjoubi NN, Porquet D (2000) Alpha-1-acid glycoprotein. Biochim Biophys Acta 1482(1-2):157-171

98. Kim NH et al (2005) Vascular endothelial growth factor (VEGF) and soluble VEGF receptor FLT-1 in diabetic nephropathy. Kidney Int 67(1):167-177

99. Rondbjerg AK, Omerovic E, Vestergaard H (2011) YKL-40 levels are independently associated with albuminuria in type 2 diabetes. Cardiovasc Diabetol 10:54

100. Lee JH et al (2012) Clinical implication of plasma and urine YKL-40, as a proinflammatory biomarker, on early stage of nephropathy in type 2 diabetic patients. J Diabetes Complications 26(4):308-312

101. Persson $\mathrm{F}$ et al (2012) High YKL-40 levels predict mortality in patients with type 2 diabetes. Diabetes Res Clin Pract 96(1):84-89

102. Ward NL, Dumont DJ (2002) The angiopoietins and Tie2/Tek: adding to the complexity of cardiovascular development. Semin Cell Dev Biol 13(1):19-27

103. Pena MJ et al (2015) A panel of novel biomarkers representing different disease pathways improves prediction of renal function decline in type 2 diabetes. PLoS One 10(5):e0120995

104. Iyengar SK et al (2007) Genome-wide scans for diabetic nephropathy and albuminuria in multiethnic populations: the family investigation of nephropathy and diabetes (FIND). Diabetes $56(6): 1577-1585$

105. Bell CG et al (2010) Genome-wide DNA methylation analysis for diabetic nephropathy in type 1 diabetes mellitus. BMC Med Genomics 3:33
106. Rossing $\mathrm{K}$ et al (2008) Urinary proteomics in diabetes and CKD. J Am Soc Nephrol 19(7):1283-1290

107. Merchant ML, Klein JB (2010) Proteomic discovery of diabetic nephropathy biomarkers. Adv Chronic Kidney Dis 17(6):480-486

108. Mischak $\mathrm{H}$ et al (2004) Proteomic analysis for the assessment of diabetic renal damage in humans. Clin Sci (Lond) 107(5):485-495

109. Siwy J et al (2014) Multicentre prospective validation of a urinary peptidome-based classifier for the diagnosis of type 2 diabetic nephropathy. Nephrol Dial Transplant 29(8):1563-1570

110. Zurbig P et al (2012) Urinary proteomics for early diagnosis in diabetic nephropathy. Diabetes 61(12):3304-3313

111. Roscioni SS et al (2013) A urinary peptide biomarker set predicts worsening of albuminuria in type 2 diabetes mellitus. Diabetologia 56(2):259-267

112. Zhao YY (2013) Metabolomics in chronic kidney disease. Clin Chim Acta 422:59-69

113. Zhang $\mathrm{J}$ et al (2009) Metabonomics research of diabetic nephropathy and type 2 diabetes mellitus based on UPLCoaTOF-MS system. Anal Chim Acta 650(1):16-22

114. Ambros V (2004) The functions of animal microRNAs. Nature 431(7006):350-355

115. Bartel DP (2004) MicroRNAs: genomics, biogenesis, mechanism, and function. Cell 116(2):281-297

116. Bartel DP (2009) MicroRNAs: target recognition and regulatory functions. Cell 136(2):215-233

117. Kato $\mathrm{M}$ et al (2011) A microRNA circuit mediates transforming growth factor-betal autoregulation in renal glomerular mesangial cells. Kidney Int 80(4):358-368

118. Kato $\mathrm{M}$ et al (2009) TGF-beta activates Akt kinase through a microRNA-dependent amplifying circuit targeting PTEN. Nat Cell Biol 11(7):881-889

119. Deshpande SD et al (2013) Transforming growth factor-betainduced cross talk between p53 and a microRNA in the pathogenesis of diabetic nephropathy. Diabetes 62(9):3151-3162

120. Chung AC et al (2010) miR-192 mediates TGF-beta/Smad3driven renal fibrosis. J Am Soc Nephrol 21(8):1317-1325

121. Kato $\mathrm{M}$ et al (2013) TGF-beta induces acetylation of chromatin and of Ets-1 to alleviate repression of miR-192 in diabetic nephropathy. Sci Signal 6(278):ra43

122. Qin W et al (2011) TGF-beta/Smad3 signaling promotes renal fibrosis by inhibiting miR-29. J Am Soc Nephrol 22(8):1462-1474

123. Krupa A et al (2010) Loss of MicroRNA-192 promotes fibrogenesis in diabetic nephropathy. J Am Soc Nephrol 21(3):438-447

124. Yang Y et al (2013) Urine miRNAs: potential biomarkers for monitoring progression of early stages of diabetic nephropathy. Med Hypotheses 81(2):274-278

125. Agarwal R et al (2014) A prospective study of multiple protein biomarkers to predict progression in diabetic chronic kidney disease. Nephrol Dial Transplant 29(12):2293-2302

126. Verhave JC et al (2013) Clinical value of inflammatory urinary biomarkers in overt diabetic nephropathy: a prospective study. Diabetes Res Clin Pract 101(3):333-340

127. Heerspink HJ et al (2015) Drugs meeting the molecular basis of diabetic kidney disease: bridging from molecular mechanism to personalized medicine. Nephrol Dial Transplant 30(Suppl 4):iv105-iv112

128. Hajhosseiny R et al (2014) Have we reached the limits for the treatment of diabetic nephropathy? Expert Opin Investig Drugs 23(4):511-522

129. Navarro-Gonzalez JF et al (2011) Pentoxifylline for renoprotection in diabetic nephropathy: the PREDIAN study. Rationale and basal results. J Diabetes Complications 25(5):314-319 
130. Adler SG et al (2010) Phase 1 study of anti-CTGF monoclonal antibody in patients with diabetes and microalbuminuria. Clin $\mathrm{J}$ Am Soc Nephrol 5(8):1420-1428

131. RamachandraRao SP et al (2009) Pirfenidone is renoprotective in diabetic kidney disease. J Am Soc Nephrol 20(8):1765-1775

132. Droge W (2002) Free radicals in the physiological control of cell function. Physiol Rev 82(1):47-95

133. Scivittaro V, Ganz MB, Weiss MF (2000) AGEs induce oxidative stress and activate protein kinase C-beta(II) in neonatal mesangial cells. Am J Physiol Renal Physiol 278(4):F676-F683

134. Ruiz $\mathrm{S}$ et al (2013) Targeting the transcription factor Nrf2 to ameliorate oxidative stress and inflammation in chronic kidney disease. Kidney Int 83(6):1029-1041

135. Pergola PE et al (2011) Bardoxolone methyl and kidney function in CKD with type 2 diabetes. $\mathrm{N}$ Engl $\mathrm{J}$ Med 365(4):327-336

136. Pergola PE et al (2011) Effect of bardoxolone methyl on kidney function in patients with T2D and Stage 3b-4 CKD. Am J Nephrol 33(5):469-476

137. de Zeeuw D et al (2013) Bardoxolone methyl in type 2 diabetes and stage 4 chronic kidney disease. $\mathrm{N}$ Engl $\mathrm{J}$ Med 369(26):2492-2503

138. Rossing P (2013) Diabetic nephropathy: could problems with bardoxolone methyl have been predicted? Nat Rev Nephrol 9(3):128-130

139. Tan SM et al (2014) Derivative of bardoxolone methyl, dh404, in an inverse dose-dependent manner lessens diabetes-associated atherosclerosis and improves diabetic kidney disease. Diabetes 63(9):3091-3103

140. Aminzadeh MA et al (2014) The synthetic triterpenoid RTA dh404 (CDDO-dhTFEA) restores Nrf2 activity and attenuates oxidative stress, inflammation, and fibrosis in rats with chronic kidney disease. Xenobiotica 44(6):570-578

141. Ichikawa $T$ et al (2009) Dihydro-CDDO-trifluoroethyl amide (dh404), a novel Nrf2 activator, suppresses oxidative stress in cardiomyocytes. PLoS One 4(12):e8391

142. Tanimoto $M$ et al (2007) Effect of pyridoxamine (K-163), an inhibitor of advanced glycation end products, on type 2 diabetic nephropathy in KK-A(y)/Ta mice. Metabolism 56(2):160-167

143. Degenhardt TP et al (2002) Pyridoxamine inhibits early renal disease and dyslipidemia in the streptozotocin-diabetic rat. Kidney Int 61(3):939-950

144. Lewis EJ et al (2012) Pyridorin in type 2 diabetic nephropathy. J Am Soc Nephrol 23(1):131-136

145. Navarro-Gonzalez JF et al (2014) Effect of pentoxifylline on renal function and urinary albumin excretion in patients with diabetic kidney disease: the PREDIAN Trial. J Am Soc Nephrol 26(1):220-229

146. Fallahzadeh MK et al (2012) Effect of addition of silymarin to renin-angiotensin system inhibitors on proteinuria in type 2 diabetic patients with overt nephropathy: a randomized, doubleblind, placebo-controlled trial. Am J Kidney Dis 60(6):896-903

147. Sayyed SG et al (2011) An orally active chemokine receptor CCR2 antagonist prevents glomerulosclerosis and renal failure in type 2 diabetes. Kidney Int 80(1):68-78

148. Di Prospero NA et al (2014) CCR2 antagonism in patients with type 2 diabetes mellitus: a randomized, placebo-controlled study. Diabetes Obes Metab 16(11):1055-1064

149. Hocher B et al (2001) Effects of endothelin receptor antagonists on the progression of diabetic nephropathy. Nephron 87(2):161-169

150. Kohan DE et al (2014) Long-term study of patients with type 2 diabetes and moderate renal impairment shows that dapagliflozin reduces weight and blood pressure but does not improve glycemic control. Kidney Int 85(4):962-971

151. Andress DL et al (2012) Clinical efficacy of the selective endothelin A receptor antagonist, atrasentan, in patients with diabetes and chronic kidney disease (CKD). Life Sci 91(13-14):739-742

152. Parvanova A et al (2013) Effect on blood pressure of combined inhibition of endothelin-converting enzyme and neutral endopeptidase with daglutril in patients with type 2 diabetes who have albuminuria: a randomised, crossover, double-blind, placebo-controlled trial. Lancet Diabetes Endocrinol 1(1):19-27

153. Sharma K et al (2011) Pirfenidone for diabetic nephropathy. J Am Soc Nephrol 22(6):1144-1151

154. Riser BL et al (2000) Regulation of connective tissue growth factor activity in cultured rat mesangial cells and its expression in experimental diabetic glomerulosclerosis. J Am Soc Nephrol 11(1):25-38

155. http://www.diabetes.co.uk/news/2014/Dec/anti-tgf-beta-1-therapy-trial-for-diabetic-nephropathy-terminated-94103031.html

156. Zhang $\mathrm{Z}$ et al (2008) Renoprotective role of the vitamin D receptor in diabetic nephropathy. Kidney Int 73(2):163-171

157. Mizobuchi M et al (2007) Combination therapy with an angiotensin-converting enzyme inhibitor and a vitamin D analog suppresses the progression of renal insufficiency in uremic rats. $\mathrm{J}$ Am Soc Nephrol 18(6):1796-1806

158. Tan X, Li Y, Liu Y (2006) Paricalcitol attenuates renal interstitial fibrosis in obstructive nephropathy. J Am Soc Nephrol 17(12):3382-3393

159. de Borst MH et al (2013) Active vitamin D treatment for reduction of residual proteinuria: a systematic review. J Am Soc Nephrol 24(11):1863-1871

160. de Vriese AS et al (2001) Antibodies against vascular endothelial growth factor improve early renal dysfunction in experimental diabetes. J Am Soc Nephrol 12(5):993-1000

161. Gronbaek H et al (1998) Effect of octreotide, captopril or insulin on renal changes and UAE in long-term experimental diabetes. Kidney Int 53(1):173-180

162. Serri O et al (1991) Somatostatin analogue, octreotide, reduces increased glomerular filtration rate and kidney size in insulindependent diabetes. JAMA 265(7):888-892

163. Clemens A et al (1999) Octreotide (somatostatin analog) treatment reduces endothelial cell dysfunction in patients with diabetes mellitus. Metabolism 48(10):1236-1240

164. Tumlin JA, Galphin CM, Rovin BH (2013) Advanced diabetic nephropathy with nephrotic range proteinuria: a pilot study of the long-term efficacy of subcutaneous ACTH gel on proteinuria, progression of CKD, and urinary levels of VEGF and MCP1. J Diabetes Res 2013:489869

165. Aggarwal HK et al (2010) Evaluation of role of doxycycline (a matrix metalloproteinase inhibitor) on renal functions in patients of diabetic nephropathy. Ren Fail 32(8):941-946

166. Momeni A et al (2010) Effect of allopurinol in decreasing proteinuria in type 2 diabetic patients. Iran J Kidney Dis 4(2):128-132

167. Maahs DM et al (2013) Uric acid lowering to prevent kidney function loss in diabetes: the preventing early renal function loss (PERL) allopurinol study. Curr Diab Rep 13(4):550-559

168. Liu $P$ et al (2014) Allopurinol treatment improves renal function in patients with type 2 diabetes and asymptomatic hyperuricemia: 3-year randomized parallel-controlled study. Clin Endocrinol (Oxf) 83(4):475-482

169. Sanders LJ (2002) From Thebes to Toronto and the 21st century: an incredible journey. Diabetes Spectrum 15(1):56-60

170. Mann JF et al (2015) Diagnosis and treatment of early renal disease in patients with type 2 diabetes mellitus: what are the clinical needs? Nephrol Dial Transplant 30(Suppl 4):iv1-iv5

171. de Boer IH, D.E.R. Group (2014) Kidney disease and related findings in the diabetes control and complications trial/epidemiology of diabetes interventions and complications study. Diabetes Care 37(1):24-30 